\title{
ETHOS Y FORMALISMO DE LA CIENCIA ECONÓMICA: EL CASO DE LA TEORÍA DE LA ELECCIÓN RACIONAL
}

\author{
Christian Escobar Jiménez* \\ Pontificia Universidad Católica del Ecuador \\ cmescobar@puce.edu.ec \\ cmescogen@hotmail.es
}

\begin{abstract}
Resumen / Abstract
El presente trabajo desarrolla varios aspectos epistemológicos de la Teoría de la Elección Racional (TER). Se da una perspectiva histórica de la tradición científica occidental en la cual se inscribe la misma, ligándose profundamente a la epistemología idealista y racionalista. Se incluye también un repaso a la revisión crítica de la "racionalidad limitada" opuesta a la TER y de algunos elementos de estudios en psicología aplicados a la economía que contradicen los supuestos de la racionalidad sustantiva. Por último, se hace un repaso de la pretensión de la TER de separarse de cualquier ética, dada su presunción de cientificidad, convirtiéndose, ella misma, en un poderoso ethos en el ámbito de la economía y, por consiguiente, en el político.
\end{abstract}

Palabras Clave: epistemología de la economía, elección racional, racionalidad limitada, ética en economía, formalismo en economía.

\section{ETHOS AND FORMALISM IN ECONOMICS: THE RATIONAL CHOICE THEORY}

This paper develops several epistemological aspects of the standard Rational Choice Theory (hereafter RCT). The introduction presents some essential criteria of the RCT in a social and scientific context, where the RCT stands on an artificial formalization to support its validity. Then, the core elements of the RCT are briefly outlined. Subsequently, a historical perspective of the Western scientific tradition is given, in which the RCT locates, linking deeply to the idealist and rationalist epistemology. A critical review of the bounded rationality opposed to the TER and some elements of study in psychology applied to economics that contradict the assumptions of substantive rationality are also included. Finally, a review is made on the ambition of the RCT to be separated from any ethics, given his presumption of scientificity, turning herself into a powerful ethos in the field of economics, and hence, of politics.

KEY WORDS: epistemology of economics, rational choice, bounded rationality, ethics in economics, formalism in economics.

Licenciado en Sociología, Máster en Economía por la EHESS de Francia, Máster en Relaciones Internacionales y Economía por la UASB de Ecuador, Máster en Filosofía de la Ciencia por la UNED de España. Doctorante en Filosofía de la Ciencia en la Universidad de Compostela España y en Estética y Música en la Universidad de París VIII de Francia. Becario del Estado ecuatoriano, docente universitario y colaborador en políticas públicas del Estado ecuatoriano. 


\section{Introducción}

RA La TER se articula como una teoría de estructura formal, basada en una serie de axiomas lógicos y matemáticos que nos dicen cómo los agentes económicos procesan la información y toman decisiones. Si bien puede existir una gran y compleja coherencia interna en la teoría en cuanto tal, el problema de la elección estándar va mucho más allá de su pertinencia formal; pues ésta pretende conjugar los aspectos predictivos y descriptivos de una ley científica, así como establecer patrones de normatividad (cómo deberían comportarse los agentes), para tomar la mejor decisión posible dentro de una acepción dada de racionalidad. Sus límites formales se disuelven en la medida en la que esta explicación pretende comportar elementos de análisis sobre la "realidad socioeconómica". De esta manera, de la estructura lógica formal se transita hacia el problema de la validez y correspondencia con la realidad.

A pesar de que la TER ha sido ampliamente analizada, discutida y refutada, su presencia en la ciencia económica tiene una importancia central y es el eje de varios programas de investigación. La TER se erige como una parte del "núcleo duro" del saber económico, sobre todo en microeconomía, y encuentra sus correlativos macroeconómicos en la Teoría del Equilibrio General (Sapir 2000, p. 46). Entendiendo a la ciencia como campo social (un mercado) en el que se intercambian valores específicos y se establecen relaciones sociales de poder, fuerza y prestigio (Zamora Bonilla 2008, p. 72), la teoría de la elección racional ha logrado su consolidación dentro de este "mercado", erigiéndose como una parte central del saber social, no solo para la economía, sino para buena parte de las ciencias sociales.

Remontándonos a la conocida fórmula de Popper, una teoría puede definir su vigencia y validez en negativo. Si tal o cual teoría se constituye como la que explica mejor un conjunto complejo de fenómenos y a la vez resiste a la falsación, se convierte en una teoría válida y vigente hasta su refutación definitiva. Por ende, la pregunta que entra en juego es: ¿Por qué si la teoría estándar de la decisión cuenta con una enorme cantidad de información que la refuta, tiene tal vigor en el campo de la comunidad científica en economía?

Una primera respuesta podría ir por el lado del mismo Popper. En las ciencias sociales, el carácter situacional de los agentes (ya asumido por Popper como diferente al agente invariable de la TER) dificulta la labor de abstracción teórica y posterior comprobación (Zamora Bonilla $a$ ). Pero claro, al parecer esto solo dificulta la comprobación, pero no la posibilidad (casi arbitraria) de construir las teorías en las ciencias sociales. Por otro lado, esta misma fortaleza está asociada a la manera abstracta en la que está concebida la teoría. En su "Metodología de la Economía Positiva", Milton Friedman nos aclara que lo importante de la economía es si logra o no predecir los eventos de su objeto de estudio y no el "realismo" de sus supuestos (Friedman 1966, p. 5). Lo importante aquí no es la validez, sino la "normatividad" y la predictividad de la teoría. En consecuencia, normatividad y predictividad se juntan para constituir una teoría que no explica los fenómenos sino cómo debemos comportarnos. 
Para ello, la modelización formal parecería ser el mejor camino. Dado el problema del "realismo" de los supuestos para toda teoría, unas son mejores que otras si la construcción de los supuestos encaja mejor para los objetivos que se persigue (Friedman 1966, p. 42). Por supuesto, esta es una forma casi tautológica de concebir las ciencias, en el intento de no encasillarse en un realismo imposible (entendido de una forma bastante ingenua), se construyen teorías basadas solo en axiomas formales. Si se asume la imposibilidad del "conocimiento real" desde lo empírico, los modelos aparecen como mejores, si estos se siguen desde los objetivos (¿conclusiones?) ${ }^{1}$.

Aquí cabe destacar la forma en la que se ha abordado este problema. En primer lugar, de acuerdo a la lógica de la ciencia, toda teoría o sistema de conocimiento debe ser demostrado para sostenerse (ya sea formal o empíricamente). Antes de erigir teorías, éstas deben basar su corpus general en los datos. Si no ha habido una validación de la teoría con los datos, por qué dedicarse a refutarla. La respuesta es simple: la presencia de la teoría en los modelos usados por la economía clásica, que, como hemos dicho, se quedan apenas en lo normativo y prescriptivo. Su concordancia con la realidad parece ser algo accesorio y descartable y no hay necesidad aparente de demostración fuera del formalismo lógico-matemático. Por todo ello, resulta curiosa la posición de falsación de una teoría que todavía no ha sido probada, pero que a pesar de ello cuenta con tanta difusión en el contexto académico y político. Una teoría que no pretende "realismo", por asumir que éste es imposible de lograr (Friedman 1966), en el caso de predecir algo, como es su objeto, lo haría más como un acto de adivinación que como deducción de los fenómenos observados.

¿Por qué, cuáles son las razones para esta vigencia? Para Bourdieu (Bourdieu 2001), el saber científico constituye un campo en el que los capitales discursivos han sido apropiados de tal manera que son restrictivos y excluyentes. Esta idea de campo científico, en el que hay un "mercado" de ideas (en el sentido en el que se intercambian libremente), sobrepasa a los límites de la construcción de la ciencia en tanto que teorías válidas (Zamora 2008, p. 72). Ninguna teoría está fuera del contexto en el que se desenvuelve. Todo saber tiene una correspondencia política, que en el caso de la TER ha sido negada por sus postulantes de una forma bastante ingenua, como si en un positivismo inicial se tratase solamente de desembarazarse de la visión metafísica del mundo para lograr la total objetividad.

En este punto, también se puede incluir la pregunta de ¿cómo una teoría que no ha conseguido pasar los filtros del "rigor científico" ha podido erigirse como ese "núcleo duro" e incontestable, a pesar de todas las objeciones? ¿Por qué un artículo más a un diálogo entre sordos? ¿Por qué se procede fuera de la lógica (por no decir de una manera absurda), demostrando la invalidez de una teoría que no se preocupa de su demostración o validez fuera de la formalidad?

En cierto sentido, los sistemas formales son autopoyéticos y no necesitan de la validez otorgada por su correspondencia con la realidad. La realidad y lo formal son 
campos paralelos, pero en la medida en la que lo formal intenta "representar" una realidad determinada, la validez de tal constructo es necesaria. La matemática aplicada puede seguir conservando su perfección formal sin tener ninguna vigencia en su aplicación.

Entonces, ¿y esta salud, esta fuerza de la teoría? Dentro del campo netamente epistemológico, la TER se inscribe dentro de tradiciones más grandes en las ciencias occidentales que le dan sentido dentro del campo científico y a la que no son ajenos otros campos del saber. Este trabajo hace una aproximación muy breve a una forma de legitimación científica ligada a las matemáticas y a la idea de que toda ciencia es un cuerpo de leyes inmutables en el sentido de la física clásica. En la física, aquel "saber de saberes" moderno, le es fundamental e indispensable una descripción extensa desde la matemática. La matemática, la ciencia formal, abstracta, pura, categórica e imperecedera por excelencia, en sí misma, puede justificar por sí misma, al parecer, esta carencia de realismo.

Por otra parte, para que se pueda formalizar al individuo, habrá que dotarlo de unos contenidos mínimos que permitan este procedimiento. Este trabajo también aborda con brevedad el problema del homo oeconomicus, tras el cual subyace una configuración ontológica del individuo, cuyo contexto social halla eco en el mercantilismo y la modernidad capitalista. Como diría Hegel en su Filosofía de la Historia, "nadie es más que su tiempo, en el mejor de los casos será su tiempo". Aquí, el problema ya puede ser dimensionado de otra manera ¿Por qué considerar estas formas específicas como ahistóricas e inmutables? Quizá porque esta formulación es necesaria para la abstracción. Poco importa si antecede o se "ajusta" a los objetivos que persiguen los clásicos y neoclásicos, pero se lo puede hallar allí, en aquel lugar inmóvil y sin tiempo desde el cual se pueden edificar unos pocos supuestos para constituir un hombre unilineal, un rational fool, como diría Sen, y cuyos constitutivos permiten delinear un sujeto codificable para ser aprehendido por la formalización matemática. Es necesaria esta reducción para dar paso a esta formalización.

\section{Una breve definición de la elección racional}

Álvarez define a la TER por la conjunción de la triada: maximización de los beneficios, equilibrio de mercado $^{2}$ y preferencias estables (Álvarez 2009, p. 119). A esto se puede añadir la necesidad del acceso y uso de información completa en la toma de decisiones y la búsqueda de una simplicidad formal en la metodología.

El marco utilitario. - La teoría clásica de la elección supone que la condición necesaria para que una elección sea racional es que el agente siempre busque maximizar su utilidad o beneficios usando la menor cantidad de recursos posibles. El modelo general

2 Con respecto al equilibrio, las posiciones son más ambivalentes. La teoría de juegos, por ejemplo, continúa con el programa de la elección racional, pero en un marco en el equilibrio cobra una dimensión diferente al de beneficios comunes e iguales. 
es una combinación del modelo Van Neumann- Morgenstern con la estructura del Teorema de Bayes para la toma de decisiones por análisis de probabilidad (Kahneman 2003, p. 167).

Información completa. - Todos los agentes tienen el mismo acceso a la información, pero lo más importante es que todos los agentes la procesan de la misma forma. Existe la presunción de un agente modélico y uniforme, con capacidades de cálculo similares y siempre lógicas sobre la base de la maximización de la utilidad personal. El proceso de elección es similar y universal. Los contextos no determinan la forma de relación del agente con su información, fuera de la búsqueda para cada caso de elección. Cualquier error de cálculo es concebido como una irracionalidad o una externalidad del proceso de elección.

Como se ha dicho, tomando la información disponible (invariable para todos los agentes), cada agente escoge siempre lo mejor para sí mismo. Las preferencias son racionales si son transitivas y completas. Todo este proceso en un marco de información completa (Álvarez 2001, p. 160).

Ahora bien, con respecto al marco de información completa, las decisiones en contextos de certeza, riesgo e incertitud sustituyen la información por las capacidades computacionales de los agentes en tomas de decisiones. El largo proceso del conocimiento sobre el riesgo, que se inicia con Pascal o Bernulli, cobra nuevas dimensiones con la teoría estándar de la decisión, pues no se asume como la "mejor" alternativa de acuerdo a fines, sino como la "esperada", por ser esta racional.

Se habla de certeza, cuando la decisión tomada por el agente tiene un único resultado conocido previamente. Se habla de riesgo cuando existen varias posibilidades conocidas por el agente y éstas son probabilizables. Hablamos de incertitud si al menos alguno de estas probabilidades es desconocida para él (Harsanyi 1977, p. 22). En el marco de la teoría económica clásica, tan solo el marco de certitud era concebido, mientras había todo un análisis del riesgo que no había sido incorporado. La TER incorporará estos elementos y los potenciará en sus modelos.

En el ámbito de la elección, habrá un proceso secuencial y consistente en el que se desarrollará la elección. Este proceso se conoce como preferencias y su condición necesaria es una información completa y siempre accesible.

Se pueden resumir los aspectos centrales de las preferencias reveladas (elemento central de la teoría), de la siguiente forma:

Si se tiene en cuenta un conjunto $X$ de alternativas posibles que se excluyen mutuamente, dentro de este conjunto, habrá preferencias de unas por sobre otras. Así, en una función de elección, dentro de un conjunto posible existen al menos dos elementos y después entre estos elementos se mantiene una relación consistente de preferencia.

Sobre estos elementos se establece una relación de preferencia. En una relación $X$ (subconjunto $\mathrm{X} \times \mathrm{X}$ ), una relación es completa y transitiva. $\mathrm{Si}, x, y, z$ pertenecen a $X$ y si $x$ es preferible a $y$ e $y$ a $z$, siempre $x$ será preferible a $z$. De otra forma, habría una inconsistencia en la elección. Se establece una función de elección 
siempre y cuando haya riesgo y no incertitud; es decir que el conjunto sea finito (Izmalkov - Yildiz 2001).

Por otra parte, hay una relación ordinal entre los elementos, pues existe una función de utilidad. El modelo funciona de manera canónica, es decir, en referencia a un elemento estándar que dé pauta a la elección. De acuerdo con Von Neumann, una relación es ordinal si los elementos son transitivos (subconjuntos) y si cumplen la ley de tricotomía ${ }^{3}$. Por ello, siempre debe haber la posibilidad de "compararlos". También se establece una relación cardinal cuando pueden ser cuantificables y hay una posición probabilizable de acuerdo a la relación de utilidad apuesta/riesgo en la elección ${ }^{4}$.

\section{Las preferencias históricas de la elección racional}

La célebre parábola de la caverna de Platón nos pone en alerta sobre los peligros de conocer el mundo a través de nuestros sentidos y la perfección inmarcesible que se halla detrás de las ideas. Platón propone una división entre mundo sensible y mundo inteligible, partición que no solo marca una diferencia de orden ontológica sobre lo que puede ser aprehendido, sino una significación axiológica de lo que "debe" ser aprendido -o más bien descubierto. El mundo externo comporta apenas un contenido accidental de las formas innatas e imperecederas, como las ideas o las formas geométricas, por ejemplo (Platón 1988: 339-342). Son estas formas las que nos permiten comprender el mundo que nos rodea. Por tanto, el mundo verdadero es el inteligible, el de las ideas, el mismo que puede ser deformado por el mundo fantasmagórico que se nos presenta a través de los sentidos. Así visto, la corroboración de las ideas con la realidad, a lo mucho, implica una afirmación defectuosa del mundo inteligible (Platón 1988: 342). Así visto, en lo posterior, de acuerdo a la tradición platónica, ¿cuál sería el sentido de la experimentación en este ámbito de cosas?

Generalmente, nos remontamos a la imagen del famoso cuadro de Rafael para referirnos a la diferencia entre la concepción platónica-idealista y la acepción aristotélica del mundo: un Platón anciano y robusto con el dedo índice de su mano derecha apunta hacia el cielo - hacia lo ideal y perfecto- y un Aristóteles, joven y viril, con la palma de su mano dirige sus pensamientos hacia la tierra. Idea y materia oponiéndose entre sí. Mientras Platón propone esta división del mundo aparente y el de las ideas, Aristóteles se opone a la visión idealista de su maestro e intenta devolverle al mundo su unicidad.

En la ley de tricotomía, las posibilidades entre 2 elementos son solo 3: $x<y, y<0 x=y$. Las variantes se introducen sobre estas bases. Por supuesto que existen variantes que enriquecen el núcleo central expuesto, también se debe considerar que en la teoría de juegos, basada en este modelo central, las formas de equilibrio varían también. Se puede citar al teorema de Arrow como ejemplo. Éste busca solventar los problemas de la intransitividad en las preferencias electorales introduciendo nuevos elementos a la base presentada. 
Siempre resulta arduo y complejo hablar de "legado griego" en campos específicos y definidos del saber, pero se puede comprender el sentido contemplativo que el saber cobraba en las posturas de platónicos y aristotélicos, pese a sus diferencias. Por un lado, el saber no es únicamente un medio de apropiación del mundo para su transformación -definido como utilidad- sino como compenetración en las causas finales que lo mueven. En este sentido, hay una coincidencia entre Platón, Aristóteles y los pitagóricos. Si bien Aristóteles nos deja en su legado una taxonomía de las ciencias a través de la observación y sus objetos de estudio, las causas finales priman a la hora de explicar el mundo (Aristóteles 2007). Esta búsqueda de formas puras se repite en el círculo de Platón, en la cosmología aristotélica o en la "música de las esferas" pitagórica; y su legado al mundo occidental establece una búsqueda de las sustancias detrás de los accidentes. De las ideas y las formas por sobre la experiencia y las corrupciones propias del mundo material.

Por otro lado, como se dijo brevemente, resta hablar de la importancia que el pensamiento cobra como categoría primordial de lo humano, por sobre cualquier otra actividad utilitaria. Platón detesta a los sofistas, pues los denuncia como cazadores de "hombres" a través de falacias puramente discursivas, insostenibles como ideas puras (Platón 1871: IV 13); de la misma forma, los pitagóricos veían en ellos a una especie de mercenarios del saber, pues lo usaban para vivir. El saber en los sofistas había perdido su forma pura y contemplativa, independiente del mundo concreto. Se detesta el trabajo manual, se lo denuncia como inferior y solo acorde a la naturaleza inferior de esclavos y extranjeros (Aristóteles 1997). El saber no busca, necesariamente, un actuar sobre el mundo, busca comprenderlo, hallar las causas que nos guían. La realidad y el trabajo no tienen el mismo estatus de las altas abstracciones. Hay, por ello, una preferencia radical por las herramientas de la inteligencia. Aunque Aristóteles no le da el estatus divino que Platón o los pitagóricos le dan a las matemáticas, es en la lógica en donde se asientan las bases del "buen pensar" -que, por supuesto, nos permite producir pensamientos bellos. El conocimiento está por ello íntimamente ligado a la belleza, a la inmovilidad (Lonchamp 1992, p. 16), a la perfección y al alejamiento de la corrupción y variabilidad del mundo: y son la geometría y las matemáticas las que mejor expresan estas características. Las matemáticas no aparecen únicamente como herramienta eficaz - pues la realidad no se piensa desde estos parámetros- sino como manifestación necesaria de perfección y belleza. La utilidad que las matemáticas habían tenido en Egipto o Babilonia, para la agrimensión, la ingeniería, etc., en cierta forma queda relegada a esta idea de belleza.

Il permet de s'élever au-dessus d'un utilitarisme qui a caractérisé la naissance des techniques dans les grandes civilisations précédentes. L'essentiel devient de comprendre les faits observés, d'en rechercher les causes. Les problèmes sont étudiés pour eux-mêmes et non en fonction de leur intérêt pratique. Le peu d'intérêt pour les techniques découle d'une hiérarchisation des taches. Le travail intellectuel est considéré comme une tache noble, contrairement du travail manuel. Utiliser la raison pour inventer de belles démonstrations et progresser ainsi de vérités en vérités procure un plaisir intellectuel auquel les Grecs furent particulièrement 
sensibles. Ce plaisir explique le gout très répandu pour la mathématique (Lonchamp 1992, p. 17) ${ }^{5}$ - Ver traducción.

Este paradigma (en términos de Kuhn) será una constante dentro del idealismo y el racionalismo en la modernidad. El humanismo rehabilita la categoría del conocimiento como objeto máximo de lo humano; mientras las grandes revoluciones de la mecánica clásica y la astronomía aportan elementos fundamentales que luego se sintetizarán en la ciencia en la modernidad (Lonchamp 1992, pp. 94-116); a saber: la observación empírica y la matemática como herramienta de explicación y modelización de lo observable en la naturaleza. De allí parte la posibilidad de establecer leyes inmutables que expliquen el funcionamiento del mundo. La física, y en particular la mecánica clásica, estructurarán la forma en la que el mundo debe ser descubierto y explicado. Detrás de los fenómenos habitan leyes que pueden ser reducidas a formas.

En su célebre "Crítica de la Razón Pura”, Kant hará una síntesis sin parangón sobre las categorías que guían la razón pura, con el agregado de la necesidad de un contenido, pues el mundo en la modernidad está listo para ser transformado. La razón es vacía sin la experiencia (juicios a posteriori), pero la experiencia no nos dice nada sin las formas puras e innatas (juicios a priori) que las preceden y confieren sentido (Kant 2005, pp. 28-30). Se llega a un punto de inflexión determinante para el estatus científico, tal como el racionalismo cartesiano proponía; para Kant, una ciencia lo es si puede ser formalizada de forma matemática. Kant reconoce en Aristóteles esta forma imperecedera de la razón, pues mientras las ciencias han avanzado en sus observaciones, la lógica halló ya plena forma desde el estagirita (Kant 2005, p. 12).

Este legado modélico de la física a las ciencias no es una cuestión que afecte directamente a la denominada "física social" o a las pretensiones del positivismo; el modelo es una forma particular de entender el mundo y de legitimación matemática de este entendimiento. El famoso biólogo Ernst Mayr decía que la presunción kantiana de lo que debía ser una ciencia (explicada desde las matemáticas) es algo que había colocado a la biología a medio camino entre las ciencias duras - con su pureza nomotética y matemática-y las ciencias sociales, siempre ligadas a la especulación filosófica y sin el rigor de las abstracciones formales (Mayr 2006).

Así, los problemas de las ciencias sociales, tanto como su "mundanidad”, deben ser superados. La economía ha querido llegar a ello desde las matemáticas, superar los debates políticos y los sesgos del observador a través de la verdad matemática. Claro, aquí nadie discute la posible pertinencia y el gran rol que las matemáticas han jugado en

5 Esto permitió superar un utilitarismo que caracterizó el nacimiento de la técnica en las grandes civilizaciones precedentes. Lo esencial deviene de comprender los hechos observados, de buscar las causas. Los problemas son estudiados por su propio interés y no en función de su interés práctico. El trabajo intelectual es considerado como una tarea noble, contrariamente al trabajo manual. Utilizar la razón para inventar bellas demostraciones y pasar de verdad a verdad procura un placer intelectual al cual los griegos fueron particularmente sensibles. Este placer explica el gusto tan expandido por las matemáticas. 
el desarrollo científico moderno, se discute la validez de los modelos matemáticos, su relación con la verdad, que siguiendo la aseveración de Friedman, parece innecesaria. Solo así se puede entender la pretensión de la econometría que se define, en la mayoría de los manuales, como un programa de investigación fuera de los debates políticos e ideológicos que han impregnado a la economía política, tan venida a menos.

\section{El programa del individualismo metodológico}

El programa de investigación detrás del homo oeconomicus es el propio de la teoría política liberal y el surgimiento de las libertades individuales, tanto como de la economía mercantilista e industrial y de la libre movilidad del trabajo. Sus postulados son propios de una época particular y atraviesan una manera específica de entender la sociedad como suma de individualidades en relación o conflicto. Esto impone un ethos del cual depende la economía como forma de aplicación y entendimiento de una serie de prácticas (Weber 1987, p. 17). La definición que se da a las relaciones humanas puede definirse en negativo, como en el caso de Hobbes y la necesidad ineluctable del Estado para resolver los conflictos individuales; o como forma de conciliación de los intereses individuales en los colectivos, como en Bentham, Smith o Stuart Mill. Es en esta conciliación de los intereses individuales en donde también se asienta la noción de equilibrio, la misma que ya atraviesa de forma menos sistemática y con diferentes matices el trabajo de la escuela clásica de la economía, y que desembocará en el trabajo germinal de Walras ${ }^{6}$.

¿Cómo se logra la consolidación del programa individualista en la economía? En este punto, resulta importante entender la diferencia que persiste entre una noción deductiva del equilibrio, como en el caso de la economía clásica -de la que se infiere una confluencia de las preferencias individuales-, y un programa metodológicamente individualista, que parte de las preferencias individuales para construir una teoría general de la sociedad. En última instancia, como puede verse, la relación es inversa y aunque se comparten preceptos y conclusiones, no se puede equiparar la visión de la ciencia económica clásica (como una ciencia de la sociedad) a la teoría de la elección racional (como una ciencia de las decisiones individuales).

Geoffrey Hodgson define el desarrollo de la economía como una reacción al carácter mecanicista y nomotético que había cobrado la economía en la teoría clásica, en su búsqueda de establecerse como una física social. La influencia del marxismo revitalizaba el papel de la historia y las especificidades temporales para hacer frente a la ontología humana detrás del concepto de homo oeconomicus, aquel agente ahistórico, abstraído del tiempo y sin cultura específica que solo buscaba maximizar su utilidad de cara a la escasez (Hodgson 2001, p. 45).

$6 \quad$ Uno de los aspectos más importantes en los que diferirá el "Equilibrio General” de Walras con el de Smith, es la temporalidad de las decisiones, por ejemplo (Sapir 2000, p. 138). 
Después del marxismo, la revisión de las escuelas historicistas alemanas ${ }^{7}$ quiso aportar una mirada más bien histórica a la naturaleza adjudicada a los agentes. En la búsqueda de aportar datos para construir esta historia, se incorpora a la visión marxista una metodología individual de influencia darwiniana y a la vez positivista; que si bien se enfoca en las especificidades históricas y los datos concretos, no vuelve a cerrarse en la unicidad de las abstracciones, propia de la dialéctica marxista, por el rechazo a hacer teorías generales.

Del costado opuesto al historicismo, pero en la misma línea que derivará paradójicamente en una metodología individual, aparece la teoría walrasiana del equilibrio general. Una teoría en la que en realidad no hay agentes, pero que en el concepto central de equilibrio se permite una confluencia de los intereses individuales. La introducción de los conceptos de equilibrio y convergencia (por supuesto, entre los agentes individuales en el equilibrio parcial, tendiendo hacia el general), se presenta como un "coup de force" radical en la ciencia económica (aparentemente mucho más que el marxismo) e intenta dotar de realismo al concepto de "mano invisible" de Smith (Sapir 2000, p. 47).

Mientras las escuelas históricas alemanas tendrán una repercusión marginal en economía (su influencia será más marcada en campos como la sociología o en el Institucionalismo económico original), la teoría de Walras, adoptada por Marshall, tendrá un suceso notable.

En lo posterior, la escuela histórica encontrará una reacción bastante más virulenta en el trabajo del economista austríaco Carl Menger, quien no abandona el individualismo como metodología, pero le da un giro idealista de gran interés para la teoría estándar de la decisión. La base de la crítica de Menger es que no se puede confundir a la economía con la historia (vista además como la mera recopilación de datos). Para Menger, la pretensión del historicismo alemán de salvar a la economía del deductivismo clásico coloca a esta ciencia en un punto muerto, sin darle la fuerza explicativa necesaria de toda ciencia. "Even the most realistic orientation of theoretical research imaginable must accordingly operate with abstractions... The belief that empirical data are sufficient for all knowledge breaks down, because any empirical investigation depends on the non-empirical assumptions on identity" (Menger 2009, p. 83).

Así, Menger se concentrará en estas identidades, combinando realismo (datos) y esencialismo -búsqueda de una ontología humana- (Hodgson 2001, p. 83). Menger vuelve a la necesidad de crear una teoría duradera, con leyes aplicables a todo tiempo y espacio, basada en las esencias humanas; unas esencias que solo pueden partir de un análisis de la media de los datos. Menger define a la economía como la ciencia

7 Se definen dos escuelas en el historicismo económico alemán. La primera que surge en 1843, con la publicación de "Grundriss" por Wilhelm Roscher, y tenía entre sus miembros a Friedrich List, Karl Knies y Bruno Hildebrand. Después, la Nueva Escuela, con miembros como Gustav von Schmoller, Georg Friedrich Knapp; y una más joven, con Werner Sombart y Max Weber. 
que explica los actos que llevan a salvar esfuerzos individuales. Él pensaba que había instituciones humanas que son naturales y extensivas a toda época. Estas instituciones (la moneda o el Estado y las leyes, por ejemplo), siempre se construyen sobre la búsqueda de beneficios particulares. Los datos corroborarían esta aseveración y permiten elaborar una teoría sobre la esencia del comportamiento humano.

Hodgson lo define así: "The Methodenstreit was a major event in the history of economics thought and it changed the course of development of economic theory. For Menger, the Economics was no longer concerned with the study of economic system and process, but was a discipline focused on the economizing aspects of human behavior. It began the process in which economics was eventually transformed from the science of the economy to the science of choice" (Hodgson 2001, p. 93).

Esta forma de reabordar la ciencia económica conlleva no solo una visión metodológica desde el individualismo, sino que relega la importancia de las especificidades históricas (el problema temporal, en pocas palabras), propone principios universales e invariables y deviene en una ontología individual similar a la que ya había sido descrita en los casos de Bentham o Hobbes.

De allí, con los aportes de Pareto, Walras y Marshall, la economía empieza a tener una base para devenir en ciencia dura. La unificación de estos conceptos permitió la introducción del cálculo integral (Hodgson 2001). De la inspiración de Menger a la definición de Lionel Robbins de lo que es la economía, no hay un gran trecho: "The science which studies human behavior as a relationship between ends and scare means which have alternativa uses" (Robbins 1932, p. 15). Desde la escasez, la tendencia humana a maximizar los beneficios individuales aparece como algo esencial en la obra de Robbins. Por ende, la economía se erige como una ciencia del comportamiento individual, desde donde se puede explicar sustantivamente un comportamiento racional con arreglo a fines. En este estado de cosas, la conjugación de una perspectiva psicológica definida por el hombre utilitario y racional y la modelización matemática, serán vitales para el devenir de la economía y en una epistemología de uso corriente entre los economistas ${ }^{8}$.

\section{Los límites de la racionalidad}

¿En dónde se asientan las afirmaciones de estas características psicológicas que se atribuyen a los humanos? A saber, como se ha dicho, estas características son: una razón sustantiva para solventar la escasez y colocar de la mejor manera posible los recursos disponibles.

Para Herbert Simon, esta introducción de la visión psicológica de los individuos es, al menos, incompleta. En la psicología, todo acto puede ser razonable, pero no por

8 "In the opening words of his Principles, Alfred Marshall proclaimed Economics to be a psychological science" (Simon 1978, p. 1). 
ello racional en el sentido de la mejor elección con arreglo a fines (Simon 1982, p. S210). Esta idea de lo "razonable" introducida en psicología implica que todo acto humano puede ser explicado desde un marco específico que le da sentido, de esta manera, se puede hablar de una racionalidad implícita, la misma que no siempre estaría ligada al arreglo afines que caracteriza a la racionalidad substancial. Esta racionalidad substancial es "naturalizada" e inmanente, jamás comprendida de acuerdo a los sesgos o contextos en las cuales se desarrolla. Por ello, lo razonable puede ser entendido como una forma de racionalidad limitada al contexto y al agente. Así, la racionalidad de la psicología, de acuerdo con Simon, se opone a aquella económica, a aquel modelo sustantivo que ya preexiste de forma perfecta y que no se comporta con procedimientos diferentes de acuerdo a los agentes, al tiempo o al espacio. Así, en el sentido de Simon, solo somos parcialmente racionales, nuestra racionalidad está acotada y depende de la información disponible, de la limitación cognitiva y de cálculo de los agentes, tanto como al tiempo y la presión que sienten los agentes por tomar decisiones. El uso de métodos heurísticos es bastante más común de lo que parece y termina por oponer una racionalidad procedimental - contextual, limitada y sesgada-a una sustantiva-invariable y extensiva a todos los humanos (Simon 1982, p. S211).

En todo caso, Simon no entabla directamente una confrontación con la sustantivación del hombre egoísta, pero tampoco la asume como uniforme; sencillamente considera que el método para tomar decisiones que usamos los humanos está mucho más enmarcado al contexto de lo que se podría pensar en la sustantivación del hombre racional, egoísta y utilitario. La racionalidad no solo es instrumental, es limitada y a la vez expresiva. Un entendimiento más cercano a la realidad debe incorporar la integralidad de los procesos decisionales con la propia complementariedad de la forma de comprender estos procesos (Álvarez 2002, p. 14).

\section{¿Cómo comprender los procesos?}

La siguiente cita, a modo de metáfora del funcionamiento de la racionalidad, con los tejidos en biología o química, quizá permita entender mejor la forma en la que se filtra la información que se usa para la toma de decisiones:

Conviene que avancemos tres conceptos que, con todo su caudal metafórico, pueden resultar pertinentes para precisar una idea pragmático cognitiva de racionalidad. Se trata de las nociones de filtro informativo, membrana semipermeable (contextualmente selectora de información) y tejido (trama + urdimbre) de la racionalidad, nociones que nos van a permitir aplicar un tinte pragmático a la simple concepción sintáctica de la racionalidad e incluso nos facilitarán el colorear los puros contenidos semánticos.

En los tejidos aparecen variedades y estilos, entre los diversos tejidos uno bastante notable es aquel que se nos presenta como una malla o red en cuyo seno se expresan relaciones de orden parcial. El diseño adquiere diversas formas expresivas y en su realización la expresión se hace tejido racional: es parte de la acción además de un resultado de nuestra acción. Una idea básica es la de información, sin embargo, es conveniente distinguir una noción más o menos objetiva de información de 
otra que atienda a la información que resulta significativa para nosotros. Para cualquier organismo la información pertinente es aquella que él mismo es capaz de discriminar y procesar, además de que algunos organismos complejos son capaces de elaborar nueva información y actuar a partir de esos nuevos elementos. Sin excesivas precisiones, que nos forzarían a entrar en el ámbito de la teoría general de la información, solamente señalar que nos interesa atender a la variedad y precisamente a aquella que podemos distinguir (Álvarez 2002, p. 12).

Esta metáfora de filtros informativos lleva a Álvarez a pensar que si se trata de hallar conexiones en las ciencias naturales para la TER y la economía, la relación estímulo -respuesta para la economía estaría mejor explicada por esta metáfora de la química y de la biología que la relación unilineal de la mecánica clásica de la física (Álvarez 2001, p. 170).

La introducción de experimentos con el juego de ultimátum en economía tuvo gran impacto durante la década de los 80 . Los resultados son diversos, pero todos llevan a presentar los límites de la racionalidad y de la concepción básica de maximización de la utilidad individual a toda costa. Un experimento aplicado en países de bajo ingreso arrojó resultados sobre las preferencias de los individuos: un grupo importante de individuos dejó ir una suma considerable de dinero, con la sola condición de que desconocidos que los trataron de forma poco generosa, no ganaran una cantidad mayor que las que les fue propuesta a ellos (Kahneman 2003, p. 162). Este experimento contradice la linealidad mecánica con la que se concibe la maximización de la utilidad esperada. Si bien puede haber un comportamiento egoísta, éste no solo tiene el enfoque único de la maximización de la utilidad. Como se puede ver, hay un contexto de "punición del otro" muy importante.

Para comprender mejor este tipo de comportamiento, resulta importante conocer las formas en las que el proceso cognitivo actúa, mediante atajos que agilizan y liberan de la pesada deliberación del cálculo; más similares a los métodos de "ensayo y error" o al uso de las estructuras generales de la cognición social (Kimble et al. 2002, pp. 96-96). Estos procesos básicos de la cognición son los siguientes: estereotipos (percepción exagerada y simplificada de ciertos rasgos o características), esquemas (encasillamientos de los individuos de acuerdo a su pertenencia a grupos sociales más amplios: género, cultura, raza, etario, etc.), heurísticos (formas en las que los individuos consolidan la información existente y toman decisiones) (Kimble et al. 2002, p. 75). De estos elementos, los agentes se hacen una idea general del contexto y de los otros agentes para su conducta, juicios y la toma de decisiones. Funcionan como filtros de consciencia, interés y memoria para juicios posteriores. Aunque los juicios (entendidos como procesos más deliberativos y conscientes) sean lógicos, los presupuestos detrás de ellos estarán afectados por estas formas de procesar la información.

La Teoría de la Integración de la Información pretende explicar los procesos de cómo ensamblamos las diferentes partes desde las cuales concebimos el contexto y a los otros y desde allí actuamos. Por el contrario, la Teoría de la Elección Racional piensa a los agentes de forma aislada y sustancial, cuyas decisiones son bastante independientes de la interacción con otros agentes (Simon 1982, p. S211). Esta forma de entender la 
agencia lleva a Sen a aseverar que en la teoría estándar de la elección los agentes no son tales, pues sus decisiones, al parecer, al ser sustantivas no son electivas y tampoco hay interacción entre ellos (Sen 1977). Esta información es muy importante cuando se toma en cuenta la obviedad obviada de que la economía es una ciencia social y que necesariamente conlleva una interacción que cambia la perspectiva de los agentes (Kimble et al. 2002, p. 80).

La psicología social y la Teoría de la Integración de la Información han tratado de explicar ampliamente cómo las decisiones son afectadas por el contexto. Se han aplicado experimentos sobre deliberación y decisiones, usando casos judiciales, en los cuales los individuos reales que integran un tribunal, se enfrentan a decisiones sobre la inocencia o culpabilidad de un acusado en un juzgado. Estos casos paradigmáticos son claves para entender cómo los individuos que debían juzgar en casos en los que el proceso de deliberación contaba con la mayor cantidad de información posible, presentada de manera sistemática, tendían a emitir sus juicios, basándose en prejuicios y desconociendo la información. Al momento de deliberar y decidir, sus prejuicios eran usados como atajos cognitivos (Kimble et al. 2002: 75-77).

Las emociones, los esquemas, heurísticos, prejuicios, etc. forman parte constitutiva del "atajo" cognitivo. La memoria solo retiene una parte de la información que puede ser compatible con el esquema, estereotipo o heurístico usado en cada caso, por lo que existe una tendencia al sesgo (Kahneman 2002). Esta parte retenida, además, necesita de un proceso de accesibilidad más complejo, que limita incluso el aprendizaje, pero facilita la existencia cotidiana - no todas nuestras experiencias forman parte de nuestro proceso de toma de decisiones.

Dentro de la toma de decisiones, la maximización de la utilidad está atravesada por instituciones sociales, como la reciprocidad o el castigo, pues como se ha dicho, muchos agentes son capaces de renunciar a su utilidad para castigar a quienes contravienen la institucionalidad (Kahneman 2003, p. 162). Al parecer, las demandas efectivas de los agentes encuentran mejor eco en sistemas estables, lo que puede ser vulnerado por la ruptura de la institucionalidad y su presunción de neutralidad ante los intereses comunes.

Por otra parte, queda en entredicho la racionalidad entendida como proceso ordinal y lógico en el manejo de información. Por un lado, se ponen en evidencia las falencias del modelo de preferencias reveladas, explicado anteriormente y, por otro, la presunción de la información completa. Si bien los agentes pueden tener una visión exacta de lo que prefieren, en un contexto de riesgo, estas preferencias se diluyen. Al proceso de toma de decisiones que aparece como secuencialmente lógico, Tversky y Kahneman oponen tres elementos sustanciales para comprender cómo se presentan los juicios: el uso de heurísticos, la forma en la que se toma el riesgo (aversión al riesgo) y el contexto en el que los agentes interactúan (Kahneman 2002, p. 2).

La respuesta humana bajo incertitud está más acorde a la intuición como forma de procesar el conocimiento. Mientras que la razón es más lenta, serial, ardua, deliberada, poco flexible y está gobernada por reglas ajenas en parte a los agentes; los juicios intuitivos funcionan con conceptos y percepciones asentadas en el lenguaje cotidiano y son menos explícitos e intencionales. En la accesibilidad a estos elementos, 
el lenguaje juega un rol fundamental en su formación; conjugándose con la memoria semántica y la atención selectiva, los mismos que permiten fijar los heurísticos como forma procedimental de tomar decisiones (Kahneman 2002, p. 5).

La teoría de la elección racional, que parte de un modelo normativo, coincide con la pretensión de Frege de las capacidades de formalización del lenguaje para establecer un sistema lógico del cual se deriven conclusiones perfectamente acordes a las premisas. En lógica, se entiende por formalización a un lenguaje "artificial" que escapa de la intuición y es un proceso intencional y complejo. Al parecer, los agentes son capaces de esta formalización de forma inmediata -cuando las decisiones implican varias premisas (que además deben asumirse como verdaderas)- y también concluir en concordancia. El problema es que hay una indiferencia ante el problema del tiempo con el que se cuenta para esto, siempre variable, y en los recursos que se cuenta al formarnos los criterios. El modelo no toma en cuenta la cualidad semántica del lenguaje.

Así, hay pasos que se asumen siempre como completos: acceso a toda la información, formalización cierta y cálculo consistente. El modelo no contempla las asimetrías de la información y las considera o como fallas en la racionalidad (estupidez del agente) o externalidades al equilibrio (Stiglitz 2011, p. 599). Puede haber información completa y perfectamente formalizable, pero no necesariamente llevarnos a buenas decisiones. Ahora bien, los dos primeros casos, de información completa y formalizable, nos pueden conducir a modelos poco aplicados en la realidad, que se erigen como sistemas predictivos-normativos y pierden la potencia explicativa de la ciencia económica (Stiglitz 2001, p. 600). Es tal la postura normativa detrás de la TER: que las fallas en la predicción no son un problema de la capacidad explicativa o predictiva, sino que éstas son consideradas como una anomalía.

La neuroeconomía, parte del programa de investigación biológico-individual en la economía de las decisiones, en pruebas hechas con neuroimágenes en varios contextos (entre ellos, en juegos de ultimátum), muestran que las tareas cognitivas desarrolladas por el neocórtex, que son de tipo secuencial y podrían estar asociados a la elección racional, son muy limitadas a la evaluación consciente; mientras la mayoría de tareas se producen de forma automática, con un gobierno importante de otras regiones del cerebro, entre ellas el hipotálamo (la región cerebral del ciclo circadiano o de los procesos automáticos). Esto podría corroborar cierta "predisposición" cerebral a los procesos automáticos y la dificultad de sostener siempre juicios deliberativos (Gauthier 2006).

La TER no continúa únicamente su camino en solitario, a pesar de la evidencia, sobre todo concibe al hombre en solitario, aislado de sus congéneres y su tiempo (Sen 1977, pp. 322-324). No solo hay una limitación del problema de la concepción del agente y su contexto, sino además de cómo se entiende la información. La información no es un proceso lineal y dado, hay una forma específica de internalización. Si se confunden las señales exteriores como estímulos completos que provocan reacciones racionales, hay una absurda incomprensión de lo que es el proceso cognitivo. Todo discurso sobre la información que no sea un discurso sobre el conocimiento, es extremadamente simplista. La confusión entre señal e información reemplaza toda la complejidad del sistema cognitivo humano (Sapir 2000, p. 260). 
Los agentes no pueden sustraerse del efecto de clasificación en el que se ven enmarcados, el mismo que influye en su conducta o en la de los otros. El encasillamiento como forma de funcionalidad en las decisiones provoca no solo sesgos en la información, sino que también afecta el comportamiento final de los agentes. La profecía que se autocumple es una muestra clara de cómo los esquemas y estereotipos afectan a los individuos en sus actividades cotidianas. Este concepto explica cómo alguien puede verse influido por lo que se piensa de él y actuar sobre ello, pues hay una legitimación de identidad dentro del contexto (Kimble et al. 2002, p. 89). Estas formas extremadamente complejas de comportamiento y procesamiento de la información no únicamente no son tomadas en cuenta, sino que su evasión es parte de la metodología misma de la teoría. Dado que no existe una medida objetiva de las probabilidades acerca de ciertos estados de la naturaleza, una medida subjetiva de la probabilidad por parte de los individuos es necesaria. La búsqueda, por supuesto, no pasa por el realismo de la explicación, sino por la capacidad de reducción del modelo, teniendo en cuenta que la utilidad esperada es el motor que mueve la historia. La teoría basa la pertinencia de su simplicidad en la "navaja de Occam", pero como nos dice Simon, "Succinctness of statement is not the only measure of a theory's simplicity. Occam understood his rule as recommending theories that make no more assumptions than necessary to account for the phenomena (Essentia non sunt multiplicanda praeter necessitatem) (Simon 1978, pp. 345-346). Tal como la teoría del equilibrio general, la TER sacrifica el realismo e introduce una serie de elementos externos para no sacrificar el núcleo central de sus postulados (Simon 1982). Se induce a los elementos para que la teoría calce en cualquier circunstancia.

\section{Teoría, normatividad y ética}

La pretensión de la ciencia como el campo de lo neutro es insostenible. Es esta idea la que está detrás de los agentes modélicos que pueden ser abstraídos, de la matematización de modelos explicativos, de la idea de equilibrio o de la información perfecta. Se pretende, por otra parte, aportar realismo al modelo en base al incontestable egoísmo humano expresado en la maximización de la utilidad esperada. Me parece importante, en este punto, volver sobre el concepto de campo social de Bourdieu, el mismo que actúa como un mercado en el que no se intercambian, únicamente, valores en el sentido económico (Bourdieu 2001). Si bien el objeto de estudio de la ciencia económica es, entre otras cosas, el intercambio y las transacciones, la TER peca de pretensión al universalizar este tipo de valores a todo acto humano.

El contexto en el que nace la elección es la de la libertad, el proyecto mismo del liberalismo político está detrás. Un liberalismo que conjuga su posibilidad de elección con los métodos propios de la época de la razón. Pero esta forma específica del capitalismo, como lo entendió Weber, es extendida hasta la intemporalidad, el ahistoricismo y la aculturación. Todo esto implica la eliminación del ethos humano, entendido como marco del comportamiento.

Amartya Sen nos dice que existen relaciones más complejas y más básicas que las mismas del beneficio. No se trata de pensar en individuos altruistas por naturaleza, solamente contextualizar a los individuos, pues el concepto de utilidad es más amplio 
que el de los valores económicos (si es que tal cosa existe) y cuya concepción y cálculo está atravesado por aspectos morales del comportamiento. Una teoría que no incluya las posiciones particulares e incluso opuestas, no puede ser una teoría de un mundo complejo. El gran problema predictivo de la TER, es que esta complejidad es introducida ex post, por lo que la ética como supuesto y como medida de comportamiento no puede aparecer cuando la racionalidad falla, es parte integrada a ella (Sen 1977).

Los principios y valores éticos son formas de filtro de información, opuestos a la noción de estímulo - respuesta automáticas de los rational fools. Siempre hay un proceso complejo en el manejo de la señal (Sapir 2000). Detrás de él hay un agente, con volición y facultades cognitivas propias. La elección siempre supone una simpatía del elector con lo elegido, hay una forma particular de compromiso implícita, que no puede ser negada, una forma de afirmación de los principios que entra en juego (Sen 1977). Este compromiso puede o no estar vinculado a la maximización de la utilidad, pero aun cuando esté vinculado a ella, hay un conjunto de instituciones que limitan esta posibilidad.

La forma que encuentra la TER para resolver los problemas de estos valores no cuantificables es la ordinalidad de las preferencias. Pero hay contextos diversos en los que tal ordinalidad no puede presentarse. ¿Cómo entender entonces las preferencias reveladas? Hay una ambigüedad importante en este término. Una preferencia lo es por sobre otra cosa (sincronía) o ya en sí misma (diacronía). El análisis de las preferencias reveladas es modélico (nos habla de mejores preferencias), pero su descriptividad solo está asociada a lo normativo. Hablamos de preferencias lógicas, de mejores preferencias; de la mejor forma de actuar.

En este mismo sentido, volviendo a Friedman, se sacrifica el realismo (sustentado tan solo en el egoísmo), por la predictividad. Esta predictividad también conlleva una normatividad. Nos dice cómo deberíamos actuar para que un evento suceda o no, lo que lo vincula íntimamente con la economía política -la TER y el equilibrio tienen implicaciones fuertes en microeconomía- y se despliegan juicios como dogmas, sustentados sobre la cientificidad, para el accionar social.

En última instancia, el saber sistemático, dejando de ser contemplativo, pretende incidir en la realidad; pero la TER se aplica en una realidad que ella no representa y más bien nos dice "cómo debería ser la realidad", en vez de comprenderla.

La razón erigida como el campo de lo neutro, como categoría pura de la razón, elimina la valoración ética que está por detrás de los juicios, es el campo de la simulación, el campo de lo inexistente pero modélico.

Nociones como equilibrio, racionalidad, etc. están más dentro del campo del "deber ser", de lo ideológico como normatividad, más que de la explicación de fenómenos dados (Sapir 2000). La misma vigencia de la teoría se beneficia del contexto en el que se desenvuelve. El éxito dentro de la ciencia económica, parece estar más relacionado con la profecía autocumplida que con otra cosa, avalada por una institucionalidad científica que la promueve y halla engranajes de aplicación concreta, incidiendo ideológicamente en las instituciones, funcionando así como un ethos particular desde el cual actuar. 
La conducta subsecuente entre dos agentes está delimitada por la información que estos tengan sobre los otros. El efecto de clasificación (encasillar a las personas para formar un heurístico funcional) o la profecía que se autocumple (comportarse como alguien esperaría de uno) es un aspecto vital para la conducta y la toma de decisiones. Sobre todo, la profecía que se autocumple parece estar muy relacionada con el éxito de la TER, puesto que la continuidad de la misma pasa por el prestigio de sus propuestas, avaladas por una institucionalidad que la promueve ${ }^{9}$.

\section{Conclusión}

La TER no solo pretende abstraer lo humano, sino que se abstrae de lo humano. No únicamente no hay una incorporación de los sesgos en el proceso de la información, sino una importante distancia con lo ético, cultural y temporal. La TER o la noción de equilibrio, en su postulado de consistencia y transitividad, no puede explicar una sucesión de decisiones en el tiempo, por lo que el equilibrio y la transitividad han existido desde siempre o no podemos alcanzarlos de ninguna manera, pues los agentes no tienen tiempo de acción.

Los economistas neoclásicos no solo no han podido explicar buena parte de sus postulados, sino que en el postulado de simplicidad ven tal explicación como innecesaria para sus objetivos. La incorporación de trabajos como el de Kahneman o Simon, no ha podido modificar, sobre todo en microeconomía y econometría, de forma importante los postulados del modelo.

El uso de métodos y herramientas formales en lógica, matemáticas y teoría de conjuntos no es suficiente para constituir un modelo explicativo del mundo. Si bien éstas son herramientas fundamentales en la construcción de la ciencia en la modernidad, el problema de la validez es sustancial y no basta con crear modelos deductivos para explicar la realidad. A nombre de sortear los problemas de abstraer y testear lo real y concreto, ante la imposibilidad de un realismo consistente, los métodos normales no se justifican por sí solos. La economía es una ciencia fáctica y no una ciencia formal.

La TER se sitúa en un paradigma más amplio y complejo, aunque desconozca o no quiera reconocer los legados filosóficos que están detrás de su entramado.

$9 \quad$ Al respecto, cabe notar los nombres ligados al Nobel de Economía (Oficialmente Sveriges Riksbank Prize in Economic Sciences in Memorial of Aldred Nobel). Desde su comienzo en 1969, este premio ha contribuido a perpetuar el prestigio de la teoría neoclásica, dentro de la "institucionalidad científica de la economía". En la primera década del premio, al otorgarlo a Samuelson, Friedman, Kuznets, Hayek, Arrow, incide notablemente sobre la misma "conducta científica"; apenas puesta en entredicho, 10 años después con la premiación en solitario de Herbert Simon, crítico de la formulación clásica de la TER. Por otra parte, la premiación a institucionalistas, como una forma heterodoxa de economía, apenas comienza en la década de los 90 con Coase. 
Precisamente la abstracción y la formalidad del modelo como metodología, que pretende hablarnos de una desideologización del problema de la elección, está cargada de los preceptos del idealismo y del racionalismo filosófico. La TER no solo se abstrae de los problemas procedimentales y éticos en el manejo de la información, sino que en su misma normatividad funciona como un ethos de disfraces técnicos que dirigen decisiones complejas en el campo de la economía política.

\section{Referencias bibliográficas}

Álvarez J. F. (2001), “Filtros, membranas y redes: Vínculos entre ética y economía normativa", en La Fuente, M. I., Los valores de la ciencia y la cultura. León, pp. $159-176$.

(2002), "El tejido de la racionalidad acotada y expresiva", en Michael Wrigley, ed., Dialogue, Language, Rationality: A festchrift for Marcelo Dascal. Campinas.

(2009), "Elección racional y racionalidad limitada", en GarcíaBermejo, J. C., ed., Sobre la Economía y sus métodos, Vol. 30, Enciclopedia Iberoamericana de Filosofía. Madrid: Editorial Trotta y CISC, pp. 117-197.

Aristóteles (1997), Política. Madrid: Austral.

(2007), Metafísica. Madrid: Espasa Calpe.

Bourdieu Pierre (2001), Poder, Derecho y Clases Sociales. Bilbao: Desclée de Brouwer.

Friedman Milton (1966), The Methodology of Positive Economics. Essay in Positive Economics. Chicago: University Press, pp. 3-16.

Gauthier, B. I. M. (2006), Les neurosciences et l'économie : perspectives de la neuroéconomie. Univeristé du Québec à Montreal.

Harsanyi John (1977), Rational Behavior and Bargaining Equilibrium in Games and Social Situations. Cambridge University Press.

Hodgson Geoffrey (2001), How Economics Forgot the History. London: Rutledge.

Izmalkov, S. - M. Yildiz (2001), Sinopsis de la teoría de la elección. Working Paper.

Kahneman Daniel (2003), "A Psychological Perspective on Economics”, American Economic Review, Vol. 93 (2), pp. 162-168.

Kahneman Daniel (2002), Maps of Bounded Rationality: A Perspective on Intuitive Judgement and Choice. Nobel Lecture Prize.

Kant Immanuel (2005), Crítica de la razón pura. Madrid: Taurus.

Kimble Ch. et al. (2002), Psicología Social de las Américas. México: Pearson.

Lonchamp Jean-Pierre (2002), Science et croyance. Paris: Desclée de Brouwer.

Mayr Ernst (2006), ¿Por qué es única la biología? Buenos Aires: Ed. Katz.

Menger Carl (2009), Investigations into the Method of Social Sciences with Special Reference to Economics. Alabama: Institute Von Mises. 
Platón (1988), La República. Madrid: Editorial Gredos.

Robbins Lionel (1932), An Essay on Nature and Significance of Economic Science. Londres: Macmillan.

Sapir Jacques (2000), Les trous noirs de la science économique: essai sur l'impossibilité de penser le temps et l'argent. Paris: Albin Michel.

Sen Amartya (1977), "Rational Fools: A Critique of the Behavioral Foundations of Economic Theory". Philosophy and Public Affairs, Vol. 6, N 4, pp. 317 - 344.

Simon Herbert (1978), Rational Decision-Making in Business Organizations. Nobel Memorial Lecture.

(1982), "Rationality in Psichology and Economics", The Journal of Business, Vol. 59, N. 4, Part 2: The Behavioral Foundations of Economic Theory, pp. S209-S224.

Stiglitz Joseph (2011), "Rethinking in Macroeconomics: What failed, and How to Repair It", Journal of the European Economic Association 9 (4), pp. 591-645.

Weber Max (1987), Ensayos sobre sociología de la religión. Madrid: Taurus.

Zamora Bonilla J. (2008), “¿Es la ciencia un mercado de ideas?”, ArtefaCtoS, Vol. 1 $\mathrm{N} 1$, pp. $71-80$.

(2004), La economía y la filosofia popperiana de la ciencia, en W. González (ed.), Karl R. Popper: Revisión de su legado, 2004, pp. 453-486. 